\title{
Removal of Knotted Nasogastric Tube: A Literature Review and Lessons From Our Case
}

\author{
Chang Eurn Kim, $\mathrm{MD}^{1}$, In Gyu Hwang, MD, $\mathrm{PhD}^{2}$, and Hyun Jin Min, MD, $\mathrm{PhD}^{1}$ \\ Departments of ${ }^{1}$ Otorhinolaryngology-Head and Neck Surgery and ${ }^{2}$ Internal Medicine, Chung-Ang University Hospital, \\ Chung-Ang University College of Medicine, Seoul, Republic of Korea
}

\begin{abstract}
Nasogastric tube placement is a common practice, and complications are rarely reported. We recently encountered and successfully managed a knotted nasogastric tube in a chronically bedridden patient. His nasal and nasopharyngeal mucosa were exceptionally dry, and the tube was not well advanced. The attending physician had difficulty removing the tube, and unsuccessful removal attempts resulted in the patient experiencing epistaxis and respiratory discomfort. Imaging and endoscopic evaluation revealed that the tube was knotted at the choanal level, obstructing the posterior airway. We cut the tube within the middle nasal cavity and removed the knotted part through the mouth; the remaining parts of the tube were removed through the nasal cavity. Herein, we describe our case and other previously reported cases of nasogastric tube knotting to guide healthcare workers who may encounter similar scenarios.
\end{abstract}

Keywords: Intubation, gastrointestinal; Nasal cavity; Nasal mucosa; Epistaxis; Otolaryngology.

\section{INTRODUCTION}

Nasogastric tube placement is a common practice in many medical fields. It can be applied in the management of critically ill patients for enteral feeding, medication administration, or stomach decompression. ${ }^{1)}$ There are potential risks involved with nasogastric tube placement, such as aspiration from inadvertent pulmonary placement, as well as nasopharyngeal or esophageal trauma. ${ }^{2)}$ Among the potential complications associated with nasogastric tube use, knotting of the tube is rarely reported. We recently experienced a case of nasogastric tube knotting, which was resolved via simultaneous cutting through the oral and nasal routes. Herein, we review our case and other previously reported cases of nasogastric tube knotting.

Received: May 3, 2021 Revised: May 26, 2021

Accepted: May 30, 2021

Address for correspondence: Hyun Jin Min, MD, PhD, Department of Otorhinolaryngology-Head and Neck Surgery, Chung-Ang University College of Medicine, 102 Heukseok-ro, Dongjak-gu, Seoul 06973, Republic of Korea Tel: +82-2-6299-1765, Fax: +82-2-825-1765, E-mail: jjinient@cau.ac.kr

This is an Open Access article distributed under the terms of the Creative Commons Attribution Non-Commercial License (https://creativecommons.org/licenses/by$\mathrm{nc} / 4.0$ ) which permits unrestricted non-commercial use, distribution, and reproduction in any medium, provided the original work is properly cited.

\section{CASE REPORT}

We responded to an emergency otorhinolaryngology consultation request regarding a 66-year-old male with terminal colon cancer who was experiencing respiratory discomfort and epistaxis. The patient had been chronically bedridden, and a decision was made to insert a nasogastric tube for nutritional support. The attending physician made multiple attempts to insert a $12 \mathrm{~F}$ nasogastric tube over 30 minutes. However, the attending physician decided to remove the nasogastric tube after it was noted to be poorly placed, and the patient expressed pain and respiratory discomfort. However, attempts to remove the tube were met with resistance, and the patient experienced severe pain and developed epistaxis-the tube was effectively stuck. A lateral neck X-ray suggested that the nasogastric tube was rolled up at the choanal level (Fig. 1A). The otorhinolaryngology department was consulted for removal of the nasogastric tube. Flexible nasal endoscopy revealed that the nasal mucosa was exceptionally dry, and the distal part of the inserted nasogastric tube was knotted, obstructing the posterior upper airway. Oropharyngeal examination reaffirmed the extreme mucosal dryness and identified the distal part of the inserted tube in the posterior oropharynx. We decided to remove the tube via a simultaneous nasal and oral approach by cutting the midportion of the tube. The oropharyngeal portion of the tube distal to the knotted seg- 

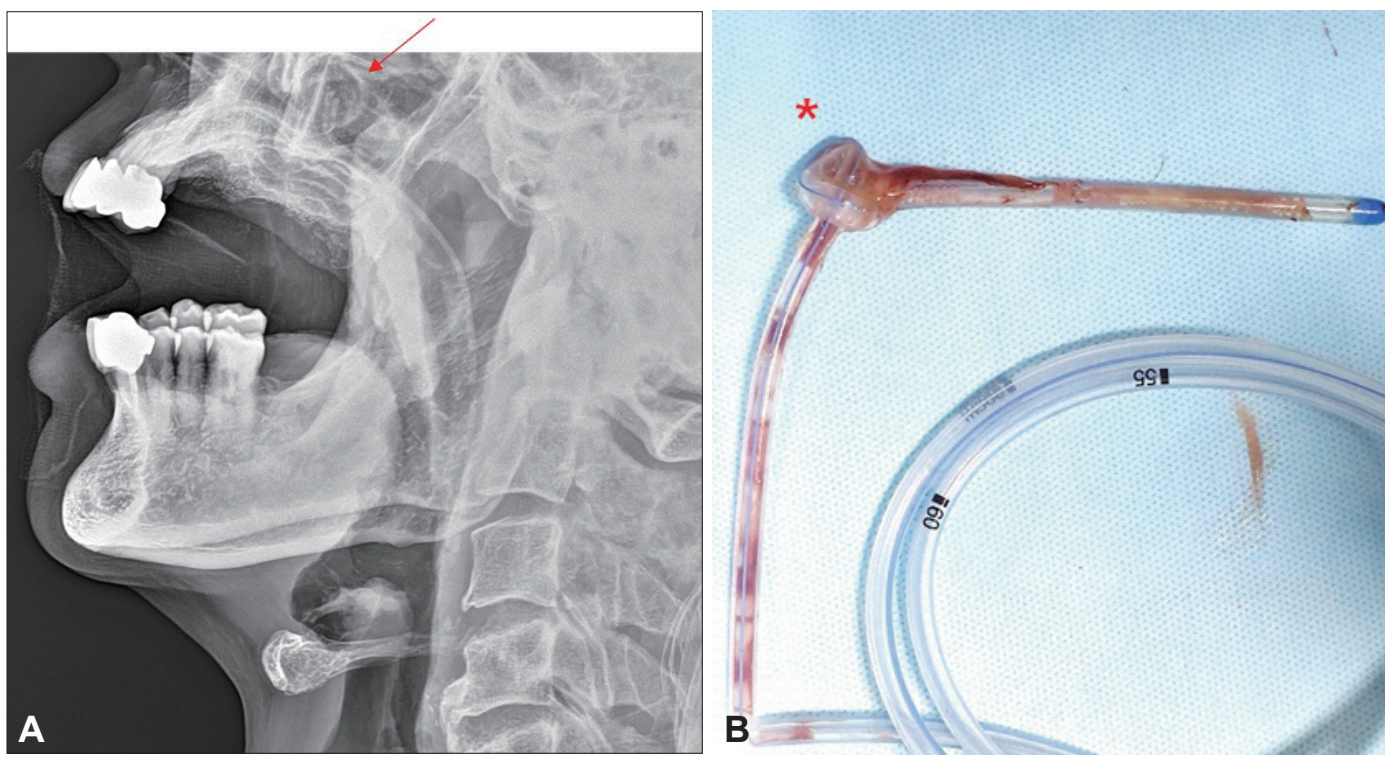

Fig. 1. Images of knotted nasogastric tube. A: Lateral neck X-ray suggesting the coiling of the nasogastric tube at the choanal level (arrow). B: Photo showing true knot formation $7 \mathrm{~cm}$ from the distal end of the nasogastric tube (asterisk).

ment was grasped using artery forceps, and the tube was cut at the level of the middle turbinate within the nasal cavity. The proximal nasal portion of the inserted tube was removed through the nasal cavity, and the knotted distal portion was removed through the mouth. After the nasogastric tube was pulled out, we confirmed that the nasogastric tube was knotted, which might have resulted from forceful insertion and retraction through dry nasal and oropharyngeal mucosa (Fig. 1B). This rare case demonstrated how forced insertion and retraction of a nasogastric tube could lead to knotting of the tube, inducing nasal complications such as pain, epistaxis, and respiratory discomfort, especially among patients with dry nasal mucosa.

This study was approved by the Institutional Review Board of Chung-Ang University College of Medicine (2105-00319365), and the requirement for informed consent was waived.

\section{DISCUSSION}

Although nasogastric tube insertion is a routine procedure, it can cause unexpected complications, including aspiration, perforation of vascular structures, pneumothorax, epistaxis, tracheal or intracranial misplacement, and a retained nasogastric tube. ${ }^{3)}$ Severe complications have rarely been reported, and the associated mortality rate has been reported to be $0.3 \%{ }^{3) 4}$ The majority of complications, including true knot formation, are due to faulty insertion of the tube. ${ }^{3)}$

Several risk factors potentially associated with nasogastric tube knot formation upon tube insertion have been reported, including the use of narrow-bore tubes, excessive tube length, small bursts of violent peristalsis, prolonged duration of tube placement. Furthermore, patients with head trauma or small gastric remnant induced by previous gastric surgery could be also risk factors of nasogastric tube knotting. ${ }^{2) 3}$ In this case, we suspect that the patient's exceptionally dry nasal and nasopharyngeal mucosa might have resisted tube advancement, leading to forceful insertion and rolling of the tube; subsequently, forceful retraction led to knotting of the tube. In those cases, we suggest that sufficient humidification of nasal mucosa and guidance of fibroscopic nasal endoscopy would be helpful in nasogastric tube insertion.

There are early signs that suggest the possibility of nasogastric tube knotting. As occurred in our case, such signs include difficulty in smooth advancement of the tube or the sensation of excessive resistance to tube removal. Unsuccessful aspiration of gastric contents and respiratory distress are other signs suggesting nasogastric knotting. Immediate bedside observations, such as epigastric auscultation findings, cough, dyspnoea, and cyanosis, should be emphasized for the early detection of nasogastric tube knotting. ${ }^{5)}$ When nasogastric tube knotting is suspected, further attempts to correctly situate the tube should be avoided. Confirmative tests, such as ultrasonography and X-ray, should be considered before attempting forced retraction of the tube to decrease the risk of true knot formation, causing more severe complications. ${ }^{6}$

Different nasogastric tube removal techniques have been reported. ${ }^{7 / 8)}$ Examples include passing a larger nasogastric tube over the knotted tube and removing them together and removing the knotted nasogastric tube under fibroscopic 
guidance. In our case, we observed that the distal part of the knotted tube was located adjacent to the posterior oropharyngeal wall; we removed the knotted portion through the mouth, and other parts of the tube were removed through the nasal cavity by cutting the tube at the level of the middle nasal cavity. As there are turbinate structures in the nasal cavity, attempts to retract the knotted portion of the tube could have caused nasal mucosal damage and massive nasal bleeding. Our approach was safe, rapid, and inexpensive, and it is feasible when the knotted portion is visible. In this method, the distal part of the tube should be secured with an instrument, such as artery forceps, before the tube is cut.

Based on our case, we suggest that, for chronically bedridden patients with excessively dry nasal and nasopharyngeal mucosa, much care should be applied during nasogastric tube insertion. Excessive resistance during tube advancement should prompt caution, and the procedure should be stopped immediately. In such cases, confirmative tests should be performed to precisely define the knotting site. After evaluating the nasal and oropharyngeal passages and confirming the position of the knot, healthcare staff can cut the tube in the middle nasal airway and safely remove the knotted portion through the mouth.

\section{Conflicts of Interest \\ The authors have no potential conflicts of interest to disclose.}

\section{Author Contributions}

Conceptualization: In Gyu Hwang, Hyun Jin Min. Data curation: Chang Eurn Kim. Formal analysis: all authors. Writing-review \& editing: Hyun Jin Min.

\section{ORCID iDs}

Chang Eurn Kim https://orcid.org/0000-0003-3995-9924

Hyun Jin Min https://orcid.org/0000-0003-3075-1350

Funding Statement

None

\section{REFERENCES}

1) Hirwa KD, Toshniwal N. Knotted nasogastric tube in the posterior nasopharynx: a case report. Qatar Med J 2016;2016(2):11.

2) Chavda V, Alhammali T, Farrant J, Naidu L, El-Rabaa S. Nasogastric tube knotting: a rare and potentially overlooked complication among healthcare professionals. BMJ Case Rep 2017;2017:bcr2017220287.

3) Ravind R, Prameela CG, Gurram BC, Dinesh M. Naughty knot: a case of nasogastric tube knotting. BMJ Case Rep 2015;2015:bcr2015209937.

4) Agarwal A, Gaur A, Sahu D, Singh PK, Pandey CK. Nasogastric tube knotting over the epiglottis: a cause of respiratory distress. Anesth Analg 2002;94(6):1659-60.

5) Arnau Alfonso JJ. Methods for determining the correct location of a nasogastric tube after its insertion in adults patients. Enferm Clin 2013;23(2):81-3.

6) Sorokin R, Gottlieb JE. Enhancing patient safety during feedingtube insertion: a review of more than 2,000 insertions. JPEN J Parenter Enteral Nutr 2006;30(5):440-5.

7) Santhanam V, Margarson M. Removal of self-knotted nasogastric tube: technical note. Int J Oral Maxillofac Surg 2008;37(4):384-5.

8) Conroy M, Wichmann K, Farkas N, Di-Nicola V. Challenging removal of a knotted nasogastric tube following insertion under general anaesthetic. BMJ Case Rep 2020;13(10):e238213. 